\title{
A STUDY ON THE POSSIBILITY TO INCREASE THE PSR E-P INSTABILITY THRESHOLD*
}

\author{
V.Danilov, J.Galambos, D.Jeon, J.Holmes, D.Olsen, ORNL SNS Project, Oak Ridge, TN \\ D.Fitzgerald, R.Macek, M.Plum, LANL LANSCE, Los Alamos, NM \\ J.Griffin, A.Burov, FNAL, Batavia, IL
}

\begin{abstract}
The Proton Storage Ring (PSR) has a fast intensitylimiting instability, which may result from an electron cloud interaction with the circulating proton beam leading to a transverse mode coupling instability. Multipacting may also be very important. A PSR beam experiment is proposed with an available 150-350 MHz RF cavity. This cavity would produce a high frequency longitudinal density ripple on the proton bunch which could reduce the e-p instability by two mechanisms: (1) a $\sim 350 \mathrm{MHz}$ ripple could clear electrons by creating an unstable parametric resonance between the electrons and protons, and (2) a $\sim 150 \mathrm{MHz}$ ripple could reduce multipacting by reducing the electron energy at the vacuum chamber.
\end{abstract}

\section{INTRODUCTION}

The LANL PSR has a fast instability that limits the proton beam intensity. A probable explanation of this instability is that there exists a large electron density in the vacuum chamber resulting in an electron interaction with the proton beam leading to a transverse mode coupling instability between the circulating protons and oscillating electrons trapped in the proton potential well. Multipacting can drastically increase the electron density, increasing the instability. A high frequency longitudinal density ripple on the proton bunch could reduce this instability by two mechanisms.

(1) The first mechanism is clearest for a coasting beam. Electrons could accumulate during beam injection in the proton potential well, and after reaching some threshold density, could generate unstable coupled oscillations between themselves and the proton beam. In this case the lighter electrons gain large amplitudes and strike the vacuum chamber wall, producing an avalanche of secondary emission SEM electrons, resulting in the instability. A high frequency, $\sim 350 \mathrm{MHz}$, variable frequency cavity could continuously clear electrons by creating a longitudinal bunch ripple. The frequency of the cavity could be adjusted to produce an unstable parametric resonance between this longitudinal bunch ripple frequency and electron oscillation frequency.

(2) The second mechanism is applicable to bunched beams. It reduces multipacting occurring on the bunch tail, which has decreasing longitudinal density. For the case of a constant longitudinal density, electrons with zero initial kinetic energy at the vacuum chamber wall oscillate across the vacuum chamber gap through the circulating beam with zero energy gain. If the longitudinal bunch density is decreasing the electrons gain energy. It is speculated that a multipacting avalanche can build on the trailing edge of the proton bunch if the energy gain of the electrons is above $50 \mathrm{eV}$ (for aluminium vacuum chamber). Instability measurements [1] show large proton beam oscillations on the bunch tail. This is evidence in favour of the multipactor effect. A lower frequency $\sim 150$ $\mathrm{MHz}$ cavity could reduce multipacting on the trailing edge of the beam bunch by reducing the kinetic energy gain of the electrons as they traverse the proton beam tail.

Combining both effects could facilitate removal of electrons from the beam vicinity without producing a secondary emission avalanche. A PSR beam experiment is being considered, with an available RF cavity to investigate these effects.

\section{PARAMETRIC RESONANCE}

Consider a coasting beam with a constant transverse density and a sinusoidal longitudinal density. The equation of motion for electron oscillations in the resulting potential well is the Mathieu equation:

$y^{\prime \prime}+(a-2 q \cdot \cos (2 t)) y=0$,

where $t$ is time in period units of the high-frequency RF cavity divided by $\pi, \mathrm{q}$ is the half amplitude of the proton beam longitudinal density variation, and a is. the squared electron tune wrt the half frequency of the RF cavity. The electron oscillation frequency is about 50 times the revolution frequency for the PSR.

It is evident that Eq. 1 has stable and unstable parameter zones. The widest unstable resonance exists when $a=1$ and in the region of $\mathrm{a}=1.0 \pm 0.1$, the parameter $q$ must be 0.05 to provide unstable electron motion. It is the equivalent of a $\pm 5 \%$ tune spread in the electron oscillation frequency. Since $q$ is 0.05 for unstable motion, the peak-to-peak amplitude of the density variation must be 0.2. The high-frequency RF should have twice the electron frequency. For the PSR

$\sqrt{a} \approx 50 \Omega, \Omega_{R F}=100 \Omega$, and $\Omega$ is the $2.8-\mathrm{MHz}$ revolution frequency.

For a bunched beam the electron stability issues are less transparent; however, the longitudinal density modulation would help make the electron motion 
unstable. Figure 1 shows the half trace modulus of the electron motion stability matrix versus intensity for their small oscillations in the proton beam potential. The RF cavity frequency is twice the electron maximum frequency for $4 \times 10^{13}$ protons per bunch and the longitudinal ripple relative amplitude is $25 \%$. For bunch intensities greater than $3 \times 10^{13}$ protons, the electrons can not be trapped in the proton potental. It is important to appreciate that a longitudinal density modulation could cause an energy increase in the electrons at the vacuum chamber wall and multipacting could occur.

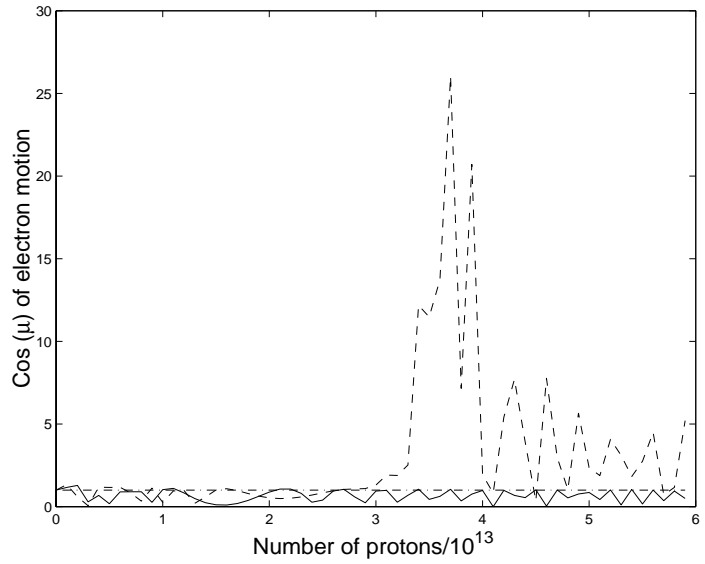

Fig. 1 Half trace modulus of electron stability matrix for electron motion versus number of protons per bunch. The dash-dot line shows the boundary of unstable motion, the solid line is a Gaussian distribution, and the dashed line is the same Gaussian with a $25 \%$ sinusoidal ripple.

\section{REDUCTION OF MULTIPACTING}

To investigate the effect of secondary emission from multipacting, a code was created that calculates 1D electron trajectories, starting from the vacuum chamber wall. After hitting the wall secondary emission electrons are produced depending on the primary initial energy. The secondary electrons oscillate with zero momentum in the proton potential since their initial energies are small in comparison with the average single-pass energy gain in the proton potential, about $100 \mathrm{eV}$. The formula for secondary emission yields from Ref. [2] was used for Al, assuming the primary electrons are normal to the surface. The peak yield is at about $400 \mathrm{eV}$.

The final result is presented as the SEM coefficient which is the natural logarithm of the average number of electrons, produced by one electron. Assuming an initial electron population of the order of $0.1 \%$ of the proton intensity, then for the total compensation of the proton charge by electrons during a single turn, the SEM needs to be about seven. That is, this initial $0.1 \%$ electron population can produce about 1000 times more electrons, $\ln (1000)=6.9$, in a single turn which is enough for the instability to occur.

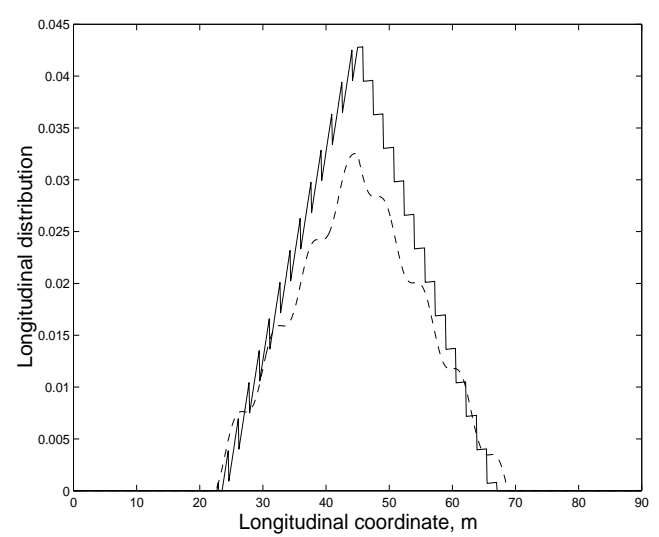

Fig. 2 Two bunch distributions used for multipacting.

Figure 2 shows two proton bunch distributions that were used to calculate secondary emission from multipacting. The upper distribution is triangular with an additional saw-tooth ripple. This is the most ideal distribution to eliminate multipacting. The lower distribution is a triangular distribution with a sinusoidal ripple. Both cases have ripple to make the biggest area with zero slopes, approaching a step function, to minimise multipacting. Figure 3 shows the SEM coefficient versus ripple amplitude with the ripple starting point at the center of the proton bunch and the end at the end of the bunch. The ripple unit is the ratio of the ripple height over the main distribution height. The dashed line presents this coefficient for the saw tooth distribution with the ripple frequency of $40 \mathrm{MHz}$. One can see that the SEM coefficient dips below zero for some optimum ripple which means that the vacuum chamber absorbs the electrons. The solid line shows this coefficient for a sinusoidal $200 \mathrm{MHz}$ ripple. It has a minimum for a ripple of about 0.03. For this minimum the SEM coefficient is 2.5 times lower than without ripple.

The numerical calculations indicate that with optimum sinusoidal the bunch intensity could be increased by about $100 \%$ and produce the same amount of electrons per turn as with no ripple. For the saw tooth optimum ripple, the electron cloud is absent for all intensities due to the large reduction of the electron energy gain during a single turn. If the bunch longitudinal tail consists of flat regions separated by a small number of density jumps, as shown as the doted line in Fig. 2, the average energy of electrons is close to zero after oscillating through the proton bunch. Hence the number of SEM electrons is close to zero. This is valid only for ripple frequencies less than the electron oscillation frequency, since for high frequency ripple the effect averages to zero. For a high frequency sinusoidal distribution the results for an arbitrary frequency are very similar to solid line in Fig. 3. The SEM coefficient has a minimum for some ripple and then grows with increasing ripple magnitude, so the RF cavity voltage needs to be controlled. 


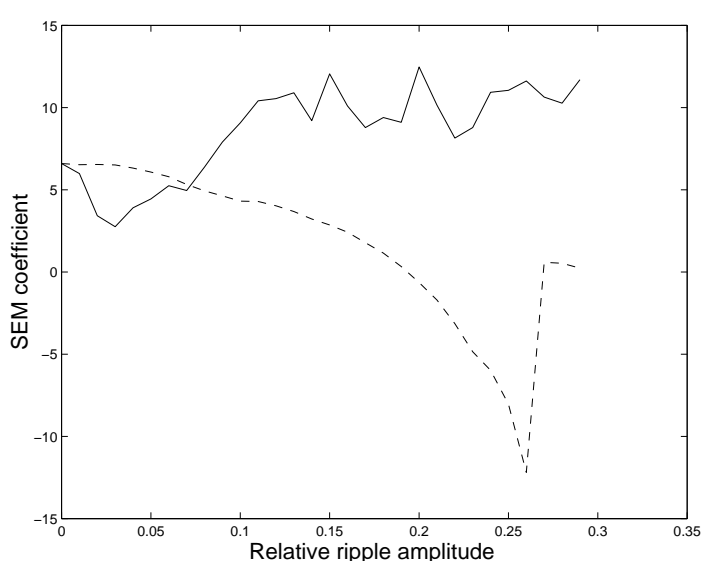

Fig. 3 The SEM coefficient versus amplitude for saw tooth (dashed line) and sinusoidal (solid line) ripples.

\section{REQUIRED CAVITY}

The longitudinal ripple can be produced with an appropriate RF voltage and frequency. The voltage can be either applied externally or generated passively by the beam. First consider an external voltage. Assume the proton longitudinal distribution in $\Delta \mathrm{E}$ and $\phi$ space has the form [3]:

$$
f(\Delta E, \phi) \propto \exp \left(-\left((\Delta E)^{2}+\frac{2 v^{2} E_{s} e V}{\eta \omega_{R F} \tau c^{2}} \cos (\phi)\right) / 2 \sigma^{2}\right)
$$

where $v$ is the proton velocity, $\mathrm{E}$ is the proton energy, $\mathrm{V}$ and $\omega$ are the RF voltage and frequency, $\eta$ is the frequency slip factor, $\tau$ is the revolution period, $\varphi$ is the synchrotron phase, and $\sigma$ is the rms energy spread. Without space charge, the amplitude $h$ of the density variation is the difference of Eq. 4 integrated over all energies $\Delta \mathrm{E}$ for the angles $\phi$ equal to zero and $\pi / 2$, divided by the $\Delta \mathrm{E}$ integrated distribution at $\phi=\pi / 2$ :

$$
h=\exp \left(\frac{v^{2} E_{s} e V}{\eta \omega_{R F} \tau c^{2} \sigma^{2}}\right)-1
$$

For a $10 \%$ amplitude modulation in the PSR, $\mathrm{h}=0.1$, and the needed RF voltage is about $40 \mathrm{kV}$, where the initial relative rms energy spread was taken equal to $10^{-3}$ and $\omega_{\mathrm{RF}}$ $=280 \mathrm{MHz}$.

Figure 4 shows that it is also possible to create a ripple at the end of injection using a passive cavity with the appropriate resonant frequency and shunt impedance. The code ORBIT [4] was used for this simulation, injecting 3 x $10^{13}$ protons, using parameters near an existing FNAL cavity. In particular, a $159-\mathrm{MHz}$ passive RF cavity was assumed with a shunt impedance of $40 \mathrm{k} \Omega$ and $\mathrm{Q}=100$. One can see flat areas with zero slopes at both bunch sides. The relative ripple amplitude is about $5 \%$. This case is applicable to the solid line of Fig. 3 which indicates that this cavity could help to reduce multipacting and increase the e-p instability threshold. This cavity does not affect the electron stability in the proton potential well since its frequency is far from twice the electron oscillation frequency. The ideal cavity system would be two or three harmonic combinations, one of which is close to twice the electron frequency in order to eliminate both multipacting and electron accumulation.

The ripple appearance in Fig. 4 is related to a slow longitudinal instability. This follows from the dependence of the ripple on the shunt impedance. For example, if one uses $60 \mathrm{k} \Omega$ for the shunt impedance, the simulated ripple would be about $50 \%$ and many protons would be lost. Consequently the RF cavity shunt impedance must be adjustable to operate close to the instability threshold.

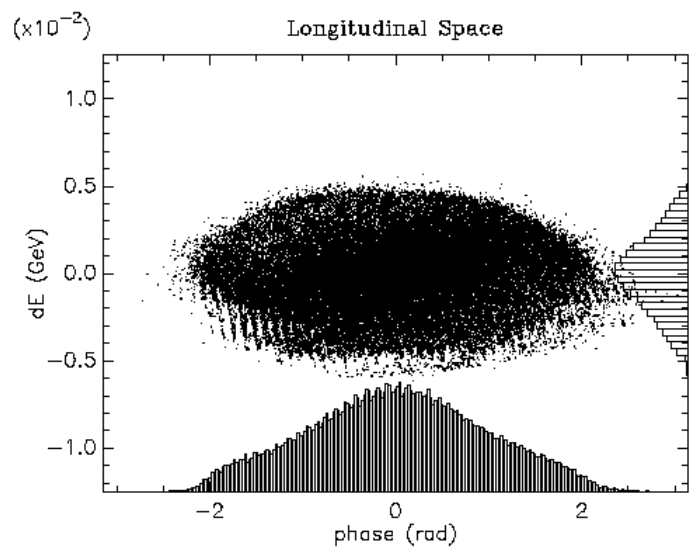

Fig. 4 Calculated PSR longitudinal distribution at the end of injection for $3 \times 10^{13}$ protons with a $159-\mathrm{MHz}$ passive, $\mathrm{Q}=100$, shunt impedance $=40 \mathrm{k} \Omega$, cavity.

*Research sponsored by the DOE, under contract no. DEAC05-96OR22464 with LMER Corp. for ORNL.

\section{REFERENCES}

[1] M.Plum, PSR Buncher Workshop, LANL, Jan 1999.

[2] F.Zimmerman, SLAC-PUB-7664 (1997).

[3] D. A. Edwards, M. J. Syphers, in An Introduction to the Physics of High Energy Accelerators, edited by M. Month (J. Wiley \& Sons, Inc., New York, 1993), p 37.

[4] J.Galambos, et al., "ORBIT", these proceedings. 